\section{Issues Emerging from a Joint Civilian-Military Nursing Program Related to Planning for and Management of a Complex Disaster \\ P. Deeny \\ University of Ulster, United Kingdom}

Objective: Recent terrorist incidents and the worldwide challenge of natural disasters highlight the need for nurses to update knowledge and skills related to disaster response and preparedness. As members of the army medical or civilian health services, nurses play a major role in disaster response. However, as of yet, they have not made an equal impact on disaster planning and preparedness. This presentation reports on an evaluation of a joint civilian-military educational program that focused on the nursing input to "planning for and management of a complex disaster". The aim of the program (and the related simulated disaster) was to provide civilian and military nurses with the necessary knowledge and skills to plan healthcare services for a complex disaster. The 36-hour simulated disaster combined a terrorist incident with an air disaster and a chemical/biological incident. This resulted in mass population displacement and the breakdown of the normal healthcare infrastructure in the Northwest of Northern Ireland.

Methods: A combined data collection approach was implemented using participant observation by those involved in delivery of the program, focus groups, and a questionnaire. The sample consisted of course participants $(n=26)$, casualty actors who were nursing students $(n=90)$, casualty welfare officers $(n=4)$, exercise control team $(n=5)$, and representatives of agencies who contributed to the program $(n=6)$.

Results: There was no marked difference in the response of civilian nurses and military nurses to the evaluation of the program. Main findings for both groups point to the requirement for ongoing support during the disaster situation. It was found that some staff are not accustomed to the stress and fatigue, and this must be considered in the planning of future training. Strategies are needed to prevent the breakdown of teamwork especially if nurses are coming from different organizations and different levels of experience. The development of "strained or aggressive interaction" with survivors may have to be accepted as a feature of the stress response and must be planned for in all nursing teams. Care of Priority 3 casualties and care of families seeking information is challenging for a healthcare system that has an "emergency care viewpoint". Complex disasters result in such issues. Other issues that were program-specific relate to risk management in relation to "moving and handling" during disasters, the role of "casualty actor" (undergraduate nursing students) and the need to expand the number of agencies involved.

Conclusion: The joint program and related disaster simulation brings up issues related to staff "stress response" in disasters and the potential for poor team functioning and poor quality of care. Pre- and post-test questionnaires should be administered to more accurately assess the changes that occurred in both groups. Overall, the evaluation confirms that nurses (both military and civilian) benefit from this "joint approach" to training and together can make a positive contribution to disaster planning and response.

Keywords: civilian; disaster; management; military; nurses; planning; response; stress

Prebosp Disast Med 2005;20(2)::87

\section{Significance and Problems of Disaster Education in a Central, Disaster Base Hospital}

Y. Tomoyasu; Y. Haraguchi; H. Nishi; T. Arai

National Hospital Disaster Medical Center, Japan

Objective: This study presents the status of and the problems encountered during the disaster courses and drills performed in a central, disaster base hospital in Japan. The aim of the courses was to expand the students' knowledge and broaden their outlook for disaster medicine and the concept of disaster itself, as well as to gain skills for dealing with disaster outcomes.

Methods: For the last nine years, disaster medical courses and disaster drills were provided regularly in the author's hospital through cooperation with the Japanese Ministry of Health, Labor, and Welfare. These courses were evaluated from the standpoint of the organizer.

Results: Disaster education courses are divided into longterm, short-term, and a lecture limited to one theme. A long-term (nearly one week) disaster education course was conducted 26 times for medical staff. The number of students participating in each course ranged from 80-120. The contents of the course varied, but a large part dealt with earthquakes. The following classification of disaster drills was proposed: (1) full-scale drill; (2) half-scale drill; (3) table-top simulation; (4) basic drill; and (5) special disaster or Nuclear-, Biological-, or Chemical- (NBC) hazard drill. Although larger-scale or full-scale disaster drills often were performed, drills of relatively small size, including tabletop simulations more frequently were repeated. From the comments of the students, the significance of disaster drills was evaluated to be effective, although several problems were pointed out including: (1) the reality of the simulation model or the scenario; (2) the reliability of medical method/process (triage, treatment, and transportation); and (3) the financial problem. Disaster medicine educaton must be established in the future.

Discussion: Ideally, all disaster medicine educational content is supplied to course participants; however, this is impossible during a week-long course. Regarding drills, a realistic, full-scale drill is useful. However, it is not easy. A tabletop exercise is recommended because of its relative simplicity. The necessity of the disaster medicine course and the adequacy of the training in real-life disasters are difficult to determine. Judging from the results of the questionnaire, participants' level of competency in disaster medicine progressed and their commitment to disaster medicine also increased. In addition, the moral or ethical viewpoint (so-called Noblesse Oblige) also should be included in the education.

Conclusions: These courses are effective for improving the level of disaster medicine expertise. However, the priority of the course contents may be reconsidered. An appropri- 
ate evaluation system for knowledge and results of the drills should be developed.

Keywords: contents; course; disaster; drills; education; Japan; outcome

Prebosp Disast Med 2005;20(2):s87-s88

\section{Evaluation of the Usefulness of the Fiberscope for Confirmation of Intubation}

Y. Koido

Nippon Medical School, Japan

Introduction: The position of the tracheal tube in tracheal intubation is confirmed by the primary and secondary confirmation methods. However, incorrect intubation (esophageal intubation) can not be detected perfectly by these methods. Prehospital tracheal intubation often results in incorrect intubation.

Objectives: A fiberscope (FS) was developed for the confirmation of intubation, and its usefulness for confirming the position of the tracheal tube was evaluated.

Methods: The position of the tracheal tube was confirmed using a FS in 30 patients who underwent tracheal intubation in the emergency room.

Results: Since one of the 30 patients showed esophageal intubation, tracheal intubation was performed 31 times. The tube was confirmed to be in the trachea in 28 of the 31 cases, and in the esophagus in one case. The position of the tube could be confirmed in $29(93.5 \%)$ of the 31 cases. In two cases, the tube position could not be confirmed because of an inadequate visual field due to airway secretion, but reintubation after tracheal aspiration allowed confirmation of the tube in the trachea. The mean time required for confirmation was $7.1 \pm 3.1$ seconds.

Discussion: At present, the apparatus used by emergency medical technicians for the determination of esophageal intubation has a sensitivity of about $70 \%$, causing many false-negative cases. Though the protocol indicates confirmation after pharyngeal re-exposure when emergency medical technicians are not confident after primary and secondary confirmation methods, this procedure is difficult. In such a situation, the FS, associated with a high accurate confirmation rate, is useful.

Keywords: accuracy; emergency medical technician (EMT); fiberscope (FS); intubation; patients; prehospital

Prebosp Disast Med 2005;20(2):s88

Neglected Protection Against Weather Influences during Mass-Casualty Incidents: Lessons Learned in Field Exercises

\section{Mattila; A. Immonen; J. Holopainen}

Department of Physiology, University of Kuopio, Finland

This report includes the authors' personal experiences from several multiple casualty incident (MCI) exercises during different seasons in Finland. The conduct of drills is an established way to test the field preparedness of health services for real-life events, in order to minimize deviations from the accepted guidelines and avoid tactical and technical mistakes.

From a trauma care perspective, the goal is to provide "severely injured" subjects with simulated procedures approximating emergency care under real circumstances. This also includes avoidance of accidental hypothermia. Even for "smart casualties", it is difficult to simulate accurately the different effects of traumas on vital signs, with an exception of body temperature. Many subjects of drills actually are cold, pale, cyanotic, and shivering, when waiting for triage, decisions, care, and transportation. Routine measures for protection against cold, which include placing blankets and aluminized plastic foil on the casualty, seem insufficient because subjects are mostly immobile and lying on the cold and moist ground. Thermal imbalance is due mainly to heat conduction to the ground. When lessons are drawn from each drill during end-of-drill discussions, it would be important to include experiences of the "smart casualties". Unofficially, "terrible cold" often is reported as the most unpleasant experience, despite wearing exceptionally warm clothing.

The last two field drills were designed to correct the neglected protection from the weather by supplying a specially designed protective covering (TelesproTM) for the emergency group to be utilized on the subjects during the exercise. Before the drill, the group members were informed as to the purpose, characteristics, and use of TelesproTM protective covering. Despite this fresh briefing, the utilization of the protection remained only partial. Therefore, much more could be done in terms of protection of casualties against weather influences, if the existence of accidental hypothermia and its negative effects becomes generally acknowledged. It also is possible to simulate cold ambience and demonstrate protective isolation needs. An ongoing systematic education and evaluation for incorporation of improved weather protection is recommended.

Keywords: analysis; field exercise; hypothermia; protective clothing; smart casualties; trauma; weather Prebosp Disast Med 2005;20(2):s88

\section{Systematic Review of Prehospital Endotracheal Intubation in Trauma Patients}

A. Sen

Manchester Royal Infirmary, United Kingdom

Background: Ventilation and prevention of aspiration are of vital importance for trauma patients. Airway is the " $\mathrm{A}$ " of "ABC" in advanced trauma life support (ATLS). Endotracheal intubation (ETI) remains the gold standard for trauma airway management. The role of ETI in out-ofhospital ATLS, however, remains controversial. Recently, an increase in mortality has been documented in association with paramedic rapid sequence intubation (RSI) of severely head-injured patients. Some experts say that early field intubation was associated with a decreased risk of fatal outcome compared with emergency department intubation, while others claim ETI confers no survival advantage over bag-and-mask ventilation (BMV) and slightly increases prehospital time. Adequate oxygenation is important to the critically injured patient to avoid secondary damage. This analysis explores benefits and harms of prehospital ETI in victims of traumatic events by a systematic review of primary studies. 